\title{
Claire Richard, Politiques de la littérature, politiques du lien chez Antoine Volodine et François Bon
}

Paris, Éditions des archives contemporaines, 2012, 224 p.

Stéphane Inkel

Université Queen's

La «transitivité » retrouvée du roman français contemporain, suivant le diagnostic de Dominique Viart, coïncide, depuis quelques années déjà, avec un élargissement des enjeux qui intéressent les chercheurs à la suite du structuralisme et de ses dérivés. On retrouve ainsi chez les critiques du contemporain une préoccupation répandue pour le politique, au sens le plus général du terme, qui fait écho aux tentatives de redéfinition nombreuses dont il est l'objet chez les philosophes continentaux, de Jacques Rancière ou de Giorgio Agamben à 
Slavoj Žižek. Les écrivains ne sont pas en reste. Si l'on a pu croire que la fin du militantisme radical, au début des années 1980, avait conduit à une mutation du politique en esthétique, comme les exemples de Pierre Michon et d'Olivier Rolin semblent vouloir l'indiquer - pensons, du premier, à la fétichisation de la Terreur en un tableau qui accapare la narration sans qu'il ne soit décrit, dans Les Onze, et dans Tigre en papier, de l'ancien militant de la Gauche prolétarienne, à la métaphore du périphérique sur lequel les protagonistes ne cessent de tourner en rond, à la périphérie du passé comme du présent -, ils sont nombreux à chercher à problématiser ce politique, au-delà de la question de l'engagement, à travers leurs œuvres. Claire Richard consacre à deux de ces écrivains les plus notoires, Antoine Volodine et François Bon, un ouvrage stimulant visant précisément à «repenser l'articulation littérature et politique » (p. 8). C'est à partir d'une commune dialectique faite de déliaison du sujet et du monde et des nouveaux rapports que ce même sujet cherche à établir à même les marges - ce que l'auteure appelle la « politique du lien » que cette articulation est pensée, ce qui ouvre un horizon de questionnements passablement large où la politique de la littérature ne se définit pas tant par le discours, bien qu'elle en fasse usage, que par une «redistribution des espaces et des temps, des places et des identités, de la parole et du bruit» (p. 10), suivant le dispositif du « partage du sensible » défini par Jacques Rancière, qui sert ici de guide pour l'analyse.

Comment établir une communauté à partir d'un geste de rupture? Quel legs pouvons-nous constituer des faillites politiques du $\mathrm{XX}^{\text {e }}$ siècle? En quel sens la littérature peut-elle être «politique» aujourd'hui? François Bon et Antoine Volodine cherchent tous deux à cerner ces questions à travers 
leur œuvre respective, selon des modalités toutefois fort différentes. Si le premier cherche à faire mémoire des lieux de plus en plus nombreux laissés en déshérence, des espaces proprement industriels à la ville, cherchant à recréer, le temps d'un livre, les liens indispensables à une situation d'interlocution, le second « oppose [au monde] un refus total » et cherche par l'univers autarcique du post-exotisme à faire « concurrence au réel» (p. 104). Or cet univers, c'est ce qui fait sa spécificité, est «entièrement déterminé par l'histoire du siècle » (p.63), ce que démontre éloquemment l'aporie où se tient le personnage post-exotique: conscience de l'échec de tout projet révolutionnaire, qui finit immanquablement par " broyer ${ }^{1}$ » ses acteurs, et celle du désastre encore plus grand de la disparition d'un tel projet. Les textes se tiennent donc dans une curieuse posture mélancolique qui cherche à rejouer les révolutions du siècle pour en garder les possibles ouverts, tout en sachant d'avance le moment irrémédiablement perdu.

Le premier mérite de l'ouvrage de Claire Richard consiste à repérer l'articulation du littéraire et du politique dans des «formes de représentation» (p.50) faites de tensions plutôt que dans les formations discursives proprement dites. À cet égard, le corpus retenu permet de mettre à l'épreuve ce choix méthodologique puisque Bon comme Volodine prennent volontiers position à travers leurs narrateurs ou le choix de leurs thématiques et se situent tous les deux à la gauche du spectre politique. Or l'essentiel se joue ailleurs, comme le montre bien Richard à propos de la «politique du lien » visible

\footnotetext{
${ }^{1}$ Le terme est de Volodine lui-même, dans un entretien accordé à Jean-Didier Wagneur dans Antoine Volodine. Fictions du politique, Écritures modernes, no 8, sous la dir. d'Anne Roche et Dominique Viart, Caen, Lettres modernes Minard, 2006, p. 236, cité par Claire Richard, p. 40.
} 
dans les romans de Bon, où il s'agit bien plus de créer des agencements, de "rétablir une continuité » (p. 83), qui fait défaut dans le réel, que de prendre position en faveur de tel ou tel collectif. Ce parti pris pour la structure des textes, qui n'exclut pas la prise en compte du hors texte, on le verra, a notamment comme avantage d'éviter, sauf exception, la paraphrase d'une poétique qui se définit elle-même, tare qui affecte en particulier la critique volodinienne, piégée d'avance par le volumineux discours de l'œuvre définissant le «postexotisme».

Divisé en quatre chapitres, l'ouvrage cherche avant tout à effectuer une synthèse du rapport au politique entretenu par le roman contemporain et ne constitue donc pas une monographie à proprement parler sur Bon et sur Volodine. Cette ambition synthétique appréciable se frappe toutefois aux limites du format (il s'agit de la version remaniée d'un mémoire de deuxième cycle), et si l'auteure trace, souvent avec vigueur, de nouvelles avenues critiques, la démonstration heuristique, elle, reste le plus souvent à faire. L'ouvrage trouve donc son utilité dans son esquisse du paysage critique actuel quant à cette question du politique qui revient avec force depuis déjà quelques années.

Le premier chapitre circonscrit d'ailleurs le champ des problèmes traités en cernant la représentation que les deux écrivains cherchent à donner du politique aujourd'hui, représentation souffrant, peut-on noter, d'un fort degré de négativité. À «l'instabilité fondamentale» (p.43) du monde contemporain décelée par les écrivains (Volodine, dans un court texte reproduit en annexe, évoque le règne actuel de la «crapulerie » et «l'étouffements des générosités élémentaires 
de gauche » [«Lettre hèle-néant», p. 219-220]), répond une forme faisant la part belle aux motifs de l'éclatement et du disjoint, à l'errance comme à la fragmentation. Alors que les deux écrivains semblent se rejoindre dans leur diagnostic, les moyens formels pour s'y attaquer ne sauraient se montrer plus éloignés. Si Bon cherche, par la mise en texte de dissensus, à répondre à la destruction du lien social qui empêche ce dernier de surgir, Volodine situe au contraire ses textes, comme on l'a vu, dans une posture «d'altérité radicale » (p. 62) par rapport au réel. Or refuser le monde et lui tourner le dos, à l'instar d'un Beckett - dont Volodine n'est peut-être pas aussi éloigné qu'on pourrait le croire - c'est en soi un geste politique d'une certaine radicalité.

À la suite de cette exposition des problèmes que le roman semble se donner pour tâche, le deuxième chapitre analyse le premier volet de la " politique de la littérature » mise de l'avant par les deux écrivains en réponse à l'atomisation sociale et politique qui imprègne la représentation. Bon cherche avant tout à rendre visibles les causalités de ces fractures le plus souvent occultées du discours social, créant du même coup de nouveaux agencements entre des registres éloignés. Volodine radicalise pour sa part les conséquences d'un état de l'histoire sous la forme d'un arrêt sur image de la catastrophe. Tous les deux, avec des poétiques extrêmement différentes, s'activent donc à faire mémoire du vingtième siècle, en particulier de ses utopies. Claire Richard y va pour l'occasion d'une analyse éclairante de la conjonction qui réunit celles d'hier et de demain chez Volodine, l'idéal révolutionnaire donnant à ses textes la physionomie d'un «tombeau qui refuse de se fermer» (p. 112). 
C'est à travers son troisième chapitre, consacré à la question de la langue et à celle du lien, que nous abordons des problèmes plus directement politiques, tant il est vrai que c'est à même la langue que se constituent des effets de communauté. Cette dialectique qui rassemble l'individuel et le collectif s'exprime chez ces deux écrivains par la problématique de la voix, à laquelle l'auteure consacre de belles analyses. Or si l'auteure reconduit ici les a priori politiques de cette poétique qu'ont en commun Bon et Volodine, il est un paradoxe qui devrait retenir notre attention. En effet, il n'y a de voix collective, chez Bon, que par une opération de « dépersonnalisation» (p. 154) dont l'écriture est porteuse par un travail de transcription / réinvention d'une parole, notamment ouvrière. Les phrases infinitives dont l'écrivain est friand participeraient ainsi d'une démarche d'effacement préalable à la poursuite de visées ayant le commun pour cible. Or, s'il est vrai que la narration, par la représentation de cette oralité, cherche à occuper une perspective le plus souvent passée sous silence - celle des prisonniers, des ouvrières, des laissés pour compte de la banlieue -, visant ainsi à replacer cette perspective «à la source du discours produit sur ell[e]» (p.135), il faudrait interroger la signature éminemment personnelle qui se dégage de ce travail de dislocation de «la syntaxe issue des représentations préexistantes» (p. 89), comme a pu le définir l'écrivain dans l'un des nombreux passages métatextuels de Daewoo. La dépersonnalisation des voix, nécessaire à l'expression du commun, mènerait donc à renforcer le dispositif « auteur».

Cette question de la singularisation d'une voix supposément collective est encore plus prégnante - et paradoxale - au sein du dispositif à la fois textuel et éditorial 
donnant corps à la «communauté post-exotique» dont Volodine se présente de plus en plus comme un simple porteparole2. Qu'elle apparaisse comme le fruit d'une communauté de prisonniers (Le Post-exotisme en dix leçons, leçon onze) ou de révolutionnaires déchus, la parole post-exotique se donne toujours à entendre comme étant collective. Or cette parole collective et le jeu des hétéronymes qu'elle entraîne constituent précisément la singularité de la signature de Volodine. Ainsi, lorsque Richard écrit que «la voix est l'élément clef de la communauté » (p. 148), on a ici l'impression que l'auteure ne fait que reprendre à son compte les énoncés méta-textuels nombreux qui parsèment l'œuvre volodinienne.

Comme on peut le constater, la visée synthétique de l'ouvrage permet d'analyser nombre de stratégies formelles utilisées par le roman contemporain afin de faire écho au politique. On peut toutefois se demander s'il était nécessaire d'ajouter une analyse du hors texte, ici abordé de manière très large, des stratégies éditoriales ou des pratiques extratextuelles des écrivains (les ateliers d'écriture de François Bon, par exemple, ou son activité sur le web) aux discours critiques qui orientent la lecture des œuvres. Le dernier chapitre, notamment lorsqu'il fait intervenir le concept de «lecture actualisante » d'Yves Citton, donne ainsi la curieuse impression de vouloir contredire certaines avancées des chapitres précédents. Car si l'ouvrage permet de circonscrire les différentes modalités par lesquelles le roman contemporain vise le politique, il devient pour le moins périlleux de suggérer

\footnotetext{
${ }^{2}$ Certaines voix post-exotiques, hétéronymes de Volodine, sont désormais signataires d'ouvrages, de Manuela Draeger à l'École des Loisirs ou aux éditions de l'Olivier à Lutz Bassmann, chez Verdier.
} 
que la lecture critique est en bonne partie responsable de la réception de Bon et Volodine en tant qu'écrivains cherchant à renouveler ces modalités. Affirmer, par exemple, que lire ces écrivains, comme le font certains critiques, avec l'aide de philosophes «politiquement marqués» comme Badiou, Agamben ou Rancière "produit le sens politique des œuvres » (p. 202. Richard souligne) contribue non seulement à prêter trop de poids à ces lectures, mais réduit aussi la portée d'une politique de la littérature par ailleurs qualifiée d'«expérimentale » (p.66) en amont, ce qui suppose un véritable projet de la part des écrivains ${ }^{3}$. Ce bémol mis à part, on saura gré au livre de Claire Richard de mettre l'accent sur ce renouvellement du discours critique quant à cette question du politique et de le faire par le moyen d'un commentaire souvent stimulant sur deux des œuvres les plus intéressantes à cet égard.

\footnotetext{
${ }^{3}$ L'un des écrivains contemporains les plus intéressants et les plus actifs à cet égard est Jean-Charles Massera, auteur de We are l'Europe et, avec Éric Arlix, du Guide du démocrate, notamment son projet de « reconnexion critique » qui vise à «opérer au cœur des outils, des dispositifs de fabrication de l'imaginaire collectif [et de] faire parler les représentations pour ouvrir des plages, des espaces, des rythmes de respiration». Voir les propositions théoriques de «It's Too Late to Say Littérature (Aujourd'hui recherche formes désespérément) », Revue Ah!, no 10, 2010, p. 40.
} 